\title{
An Indirect Measuring Method with Self-Check Function for High-Frequency Pulsating Flow Rate*
}

\author{
Tong $\mathrm{ZHAO}^{* *}$, Guangzheng PENG** \\ and Yaoming $\mathrm{XU}^{* *}$
}

\begin{abstract}
An indirect measuring method with self-check function for high-frequency pulsating flow rate is proposed, and a measuring system based on the method is set up in this paper. The system can examine by means of its self-check function whether measured results of flow rate are accurate and reliable without using any other fluid measure. ment or flowmeters. The experimental results show that the system can be applied not only to the measurement of various unsteady flow rate signals, such as high-frequency pulsating flow or even nonperiodic random flow, but can also be used as a comparison standard for evaluating other measuring methods. The research provides an important measuring means for studying the dynamic characteristics of hydraulic components or systems and for developing new instantaneous flowmeters.
\end{abstract}

Key Words: Flow Measurement, Pipe Flow, Fluctuating Pressure, Unsteady Flow, High-Frequency Pulsating Flow Rate, Self-Check

\section{Introduction}

With the development of industrial techniques, not only is it necessary to find an easy method of measuring unsteady flow rate in various flow conditions, but also it is even more of a necessity to solve how to perform an examination of its exactitude because the calibration of the measurement remains to be solved. As is well known, almost all of the current flowmeters are designed for the purpose of measuring steady flow rate, so errors not to be disregarded are apt to appear in measuring unsteady flow rate. For this reason, indirect measurements have been taken into consideration in recent years ${ }^{(1)}$.

In previous research, two methods have been presented for the measurement of unsteady flow rate in pipe flow: the center-line velocity method and the differential pressure method. The validity of both methods has been proven by experiment and analy-

* Received 5th October, 1990

** Department of Fluid Power Transmission and Con. trol, Harbin Institute of Technology, Harbin, China sis $^{(2) \sim(4)}$. However, an expensive instrument, such as the laser Doppler anemometer (LDA), is needed in the former, whereas the latter makes strict demand in the determination of the measurement distance $\Delta x$ of the differential pressure. In the case of measuring high-frequency flow rate, the distance $\Delta x$ should be as small as possible in theory in order to improve the accuracy, so special attention should be paid to obtaining reliable pressure transducers with high sensitivity because the differential pressure becomes infinitely less when $\Delta x$ is very small. In fact, in determining the distance $\Delta x$, it is difficult to satisfy the above two contradictory conditions in application ${ }^{(5)}$. In addition, because some effects caused by the change of the conditions of the measurement are unavoidable, devel opment of a measuring system with a check function is expected in order to ensure that the measured results of flow rate are accurate and reliable.

Considering the advantages and disadvantages of the above two measuring methods, an indirect measurement with self-check function for high-frequency pulsating flow rate in pipe flow is proposed and a measuring setup based on the method is also esta blished in this paper. 


\section{Nomenclature}

$a$ : Velocity of pressure wave

$D$ : Pipe diameter

$D_{n}$ : Dimensionless dissipation number $\left(=L \nu / a r_{0}{ }^{2}\right)$

$f:$ Flow oscillation frequency

$g$ : Acceleration of gravity

$H$ : Pressure head

$j:$ Imaginary unit $(=\sqrt{-1})$

$J_{0}, J_{1}, J_{2}$ : Bessel functions of first kind

$L:$ Pipe length

$P$ : Pressure signal

$P_{n}:$ Flow normalized frequency $(=2 \omega L / \pi a)$

$Q:$ Flow rate signal $\left(=V \pi D^{2} / 4\right)$

$r$ : Coordinate in radial direction

$r_{0}$ : Inside radius of pipe $r^{*}=r / r_{0}$

$s:$ Laplace operator $s^{*}=s r_{0}^{2} / \nu$

$u$ : Flow velocity in axial direction

$V$ : Mean flow velocity $V^{*}=V /\left(g r_{0}^{2} / \nu\right)$

$\Delta x$ : Distance for measuring differential pressure

$Z_{c}$ : Charaoteristic impedance

$Z_{0}:$ Impedance constant $\left(=\rho a / \pi r_{0}^{2}\right)$

$\omega:$ Angular frequency

$\nu$ : Kinematic viscosity

$\rho$ : Density of fluid

$\Gamma$ : Propagation operator

\section{Subscripts}

$\wedge$ : Laplace variable

* : Dimensionless variable

2. Basic Mathematical Expressions Relating to Fluid Variables

The Navier-Stokes equation and continuation equation for axial symmetry flow in a pipe may be written in cylindrical coordinates as follows :

$$
\begin{aligned}
& \frac{\partial^{2} \widehat{u}^{*}}{\partial r^{* 2}}+\frac{1}{r^{*}} \frac{\partial \hat{u}^{*}}{\partial r^{*}}-s^{*} \widehat{u}^{*}=\frac{\partial \hat{H}}{\partial x} \\
& Z_{0} \frac{\partial \hat{Q}}{\partial D_{n}}+s^{*} \hat{P}=0 .
\end{aligned}
$$

Regarding the mean velocity $V^{*}$ (flow rate) as the output signal, transfer functions relating to fluid variables of the same cross section in pipe flow can be derived from Eq. (1).

$$
\begin{aligned}
& \hat{V}^{*}\left(s^{*}\right)=\left\{\frac{J_{2}\left(j \sqrt{s^{*}}\right)}{J_{0}\left(j \sqrt{s^{*}} r^{*}\right)-J_{0}\left(j \sqrt{s^{*}}\right)}\right\} \hat{u}^{*}\left(r^{*}, s^{*}\right) \\
& \hat{V}^{*}\left(s^{*}\right)=\left\{\frac{2 J_{1}\left(j \sqrt{s^{*}}\right)}{\left.j \sqrt{s^{*} J_{0}\left(j \sqrt{s^{*}}\right)}-1\right\}-1} \frac{1}{s^{*}} \frac{\partial \hat{H}}{\partial x}\left(s^{*}\right)\right.
\end{aligned}
$$

Two methods based on the above transfer functions have been presented for the measurement of unsteady flow rate in pipe flow: the centerline velocity method and the differential pressure method ${ }^{(2)-(4)}$.

As mentioned before, some inconveniences exist in both methods. The differential pressure method is limited to measuring high-frequency pulsating flow rate ${ }^{(5)}$. For this reason, a method called the dualpressure method is considered in this paper.

\section{Measuring Principle}

For the fluid signals in pipe flow (Fig. 1), the pressure is easier to measure. Therefore, a measurement of flow rate, which takes pressure as the primary signal, is considered. Combining Eq. (1) with Eq. (2), a transfer relation among the pressure and the flow signals in two cross sections of a pipe can be derived ${ }^{(6)}$ :

$$
\left[\begin{array}{l}
\hat{Q}_{1} \\
\hat{Q}_{2}
\end{array}\right]=\left[\begin{array}{ll}
M_{1}\left(s^{*}\right) & -M_{2}\left(s^{*}\right) \\
M_{2}\left(s^{*}\right) & -M_{1}\left(s^{*}\right)
\end{array}\right]\left[\begin{array}{l}
\hat{P}_{1} \\
\hat{P}_{2}
\end{array}\right]
$$

where

$$
\begin{aligned}
& M_{1}\left(s^{*}\right)=\frac{\cosh \Gamma}{Z_{c} \sinh \Gamma} \\
& M_{2}\left(s^{*}\right)=\frac{1}{Z_{c} \sinh \Gamma}
\end{aligned}
$$

The distributed parameter model is required for accurate analysis in pulsating laminar flow, so that the propagation operator $\Gamma\left(s^{*}\right)$ and the characteristic impedance $Z_{c}\left(s^{*}\right)$ are of the following forms:

$$
\begin{aligned}
& \Gamma\left(s^{*}\right)=D_{n} s^{*} \sqrt{N\left(s^{*}\right)} \\
& Z_{c}\left(s^{*}\right)=Z_{0} \sqrt{N\left(s^{*}\right)} \\
& N\left(s^{*}\right)=\left\{1-\frac{2 J_{1}\left(j \sqrt{\left.s^{*}\right)}\right.}{j \sqrt{s^{*}} J_{0}\left(j \sqrt{\left.s^{*}\right)}\right.}\right\}^{-1}
\end{aligned}
$$

The block diagram based on the state equation ( 5 ) is illustrated in Fig. 2. By the use of this relationship, the flow signals $Q_{1}$, and $Q_{2}$ can be obtained from the measured pressure signals $P_{1}$ and $P_{2}$. This measurement is called the dual-pressure method.

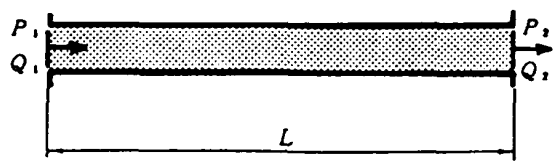

Fig. 1 Test pipe

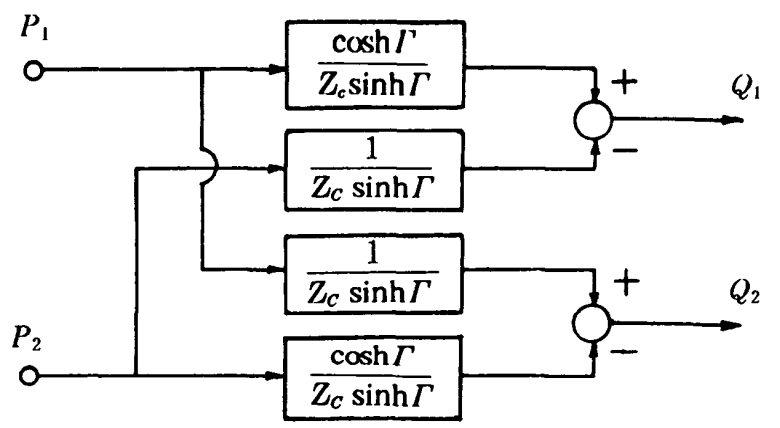

Fig. 2 Block diagram of transfer matrix 
The inherent relation between this method and the differential pressure method mentioned before will now be investigated. To use the distance $\Delta x$ for measuring the differential pressure instead of $L$ in dimensionless dissipation number $D_{n}$ in Eq. ( 8 ) and make $\Delta x$ sufficiently small, the transfer matrix Eq. (5) can be rewritten as follows because $Q_{1}=Q_{2}=Q$, $\cosh \Gamma=1$ and $\sinh \Gamma=\Gamma$ in this case :

$$
Q=\frac{\Delta x}{Z_{0} D_{n} s^{*} N\left(s^{*}\right)} \frac{\Delta P}{\Delta x} .
$$

To rearrange Eq. (11) by substituting Eq. (10) into it, the transfer function with the same form of Eq. (4) can be obtained. In the other words, there is nothing essentially different between the above two methods. The differential pressure method is no more than a specified case of the dual-pressure method. The former is based on the transfer function of fluid variables in the same cross section, while the latter is based on that in two arbitrary cross sections in pipe flow. It is quite obvious that the dual-pressure method has the following advantages:

(1) Because the method is free of the restriction to the distance $L$ measuring two pressure signals, the accuracy of flow measurement may be improved although general pressure transducers are used.

(2) Two arbitrary cross sections can be taken as measuring sections of flow rates. Therefore, it is very convenient to check the flow characteristics of other flowmeters or hydraulic elements with the aid of this method.

Nevertheless, the realization of such an indirect flow measurement requires the solving of the following problems.

\section{Accurate Approximation Models for Measure- ment}

Although Eq. (5) has established the transfer matrix relating the pressure and the flow rate signals, it is rather complicated for engineering applications because it includes Bessel functions and hyperbolic functions. Hence, $M_{1}$ and $M_{2}$ should be simplified. The accurate approximations of the matrix elements have been introduced in a preceding paper ${ }^{(7)}$, and those useful for fluid measurement are listed as follows:

$$
\begin{aligned}
& M_{1}^{\prime} \approx C_{1}\left\{\sum_{i=1}^{m} \frac{K_{i}}{s^{*}+u_{i}}+\sum_{i=1}^{n} \frac{a_{1 i} s^{*}+b_{1 i}}{s^{* 2}+2 \zeta_{i} \omega_{n i} s^{*}+\omega_{n i}^{2}}\right\} \\
& M_{2}^{\prime} \approx C_{2}\left\{\sum_{i=1}^{m} \frac{K_{i}}{s^{*}+u_{i}}+\sum_{i=1}^{n} \frac{a_{2 i} s^{*}+b_{2 i}}{s^{* 2}+2 \zeta_{i} \omega_{n i} s^{*}+\omega_{n i}^{2}}\right\} .
\end{aligned}
$$

Here $M_{1}{ }^{\prime}=Z_{0} \cdot M_{1}, M_{2}^{\prime}=Z_{0} \cdot M_{2}, C_{1}$ and $C_{2}$ represent the amending factors. The parameters of the first-order lag terms in the approximations are listed in Table 1, while the parameters of the second-order lag elements according to the dimensionless dissipation number $D_{n}$ can be calculated by the following fitting formula ${ }^{(7)}$ :

$$
\begin{aligned}
y_{j}\left(\lambda_{i}\right) & =\exp \left(A_{0}+A_{1} \ln \lambda_{i}+A_{2} \ln ^{2} \lambda_{i}\right. \\
& \left.+A_{3} \ln ^{3} \lambda_{i}+A_{4} \ln ^{4} \lambda_{i}\right) .
\end{aligned}
$$

Here, $\lambda_{i}=i / D_{n}, i$ is the ordinal number of the secondorder lag elements. $y_{i}$ are intermediate variables relating to the parameters of the second-order lag elements. Table 2 shows the coefficients $A_{k}$ of the fitting formulas. By means of the fitting formulas, $y_{1}, y_{2}, y_{3}$ and $y_{4}$ can be respectively computed. Then the parameters of the second-order lag elements in Eq. (12) and Eq. (13) can be obtained according to relationships shown in Table 3 , such as,

$$
\begin{aligned}
& \omega_{n i}=y_{1}\left(\lambda_{i}\right) \\
& \zeta_{i}=y_{2}\left(\lambda_{i}\right)
\end{aligned}
$$

For matrix element $M_{1}$ :

$$
\begin{aligned}
& a_{1 i}=y_{3}\left(\lambda_{i}\right) / D_{n} \\
& b_{1 i}=-y_{4}\left(\lambda_{i}\right) / D_{n} .
\end{aligned}
$$

For matrix element $M_{2}$ :

$$
\begin{aligned}
& a_{2 i}=(-1)^{i} y_{3}\left(\lambda_{i}\right) / D_{n} \\
& b_{2 i}=(-1)^{i+1} y_{4}\left(\lambda_{i}\right) / D_{n}
\end{aligned}
$$

\begin{tabular}{|c|c|c|c|c|}
\hline \multirow{3}{*}{$\begin{array}{l}\frac{\cosh \Gamma}{Z_{c}^{\prime} \sinh \Gamma} \\
\frac{1}{Z_{c}^{\prime} \sinh T^{\prime}}\end{array}$} & \multirow{4}{*}{$\omega_{n i}$} & \multirow{4}{*}{$\zeta_{i}$} & \multicolumn{2}{|c|}{$1 / Z_{c}^{\prime} \sinh \Gamma$} \\
\hline & & & $(-1)^{i} D_{n} a_{1}$ & $(-1)^{t+1} D_{n} b_{t}$ \\
\hline & & & \multicolumn{2}{|c|}{$\cosh \Gamma / Z_{\mathrm{c}}^{\prime} \sinh \Gamma$} \\
\hline \multirow{2}{*}{$\lambda_{i}=i / D_{n}$} & & & $D_{n} a_{i}$ & $-D_{n} b_{i}$ \\
\hline & $y_{1}(\lambda)$ & $y_{2}(\lambda)$ & $y_{3}(\lambda)$ & $y_{4}(\lambda)$ \\
\hline
\end{tabular}

In order to improve the static accuracy, it is

Table 1 Parameters of the first order lag elements in approximations of $M_{1}, M_{2}$

\begin{tabular}{|l|c|l|}
\hline$i$ & $u_{i}$ & \multicolumn{1}{|c|}{$K_{i}$ Dn } \\
\hline 1 & 5.79324 & 0.6927137 \\
2 & 30.4805 & 0.1318129 \\
3 & 77.60157 & 0.06241378 \\
4 & 196.3525 & 0.04578915 \\
5 & 552.4377 & 0.02872643 \\
6 & 1597.858 & 0.01711142 \\
7 & 4664.976 & 0.01007132 \\
8 & 13681.61 & 0.005901203 \\
9 & 40220.68 & 0.003446145 \\
10 & 118390.50 & 0.002013689 \\
\hline
\end{tabular}

Table 2 Coefficients $A_{k}$ of the fitting formulas

\begin{tabular}{|c|c|c|r|r|}
\hline \multicolumn{5}{|c|}{$y_{j}=\exp \left(\Lambda_{0}+A_{1} l n \lambda_{i}+A_{2}\left(n^{2} \lambda_{i}+A_{3} l n^{3} \lambda_{i}+A_{4} l n^{4} \lambda_{\nu}\right)\right.$} \\
\hline & $y_{1}(\lambda)$ & $y_{2}(\lambda)$ & \multicolumn{1}{c|}{$y_{3}(\lambda)$} & \multicolumn{1}{c|}{$y_{4}(\lambda)$} \\
\hline$A_{0}$ & 0.91991 & -0.203144 & 0.028976 & -1.66674 \\
$A_{1}$ & 1.0445 & -0.79793 & 0.303147 & 1.79715 \\
$A_{2}$ & $4.576 \mathrm{E}-3$ & 0.051113 & -0.056421 & -0.34934 \\
$A_{3}$ & $-1.635 \mathrm{E}-3$ & $-4.126 \mathrm{E}-3$ & $4.928 \mathrm{E}-3$ & 0.04170 \\
$A_{4}$ & $9.918 \mathrm{E}-5$ & $1.276 \mathrm{E}-4$ & $-1.663 \mathrm{E}-4$ & -0.00183 \\
\hline
\end{tabular}

Table 3 Relations of intermediate variables $y_{j}$ and parameters of the approximations 
necessary to calculate the amending factors $C_{1}$ and $C_{2}$ :

$$
\begin{aligned}
& C_{1}=\left\{8 D_{n}\left(\sum_{i=1}^{m} \frac{K_{i}}{u_{i}}+\sum_{i=1}^{n} \frac{b_{1 i}}{\omega_{n i}^{2}}\right)\right\}^{-1} \\
& C_{2}=\left\{8 D_{n}\left(\sum_{i=1}^{m} \frac{K_{i}}{u_{i}}+\sum_{i=1}^{n} \frac{b_{2 i}}{\omega_{n i}^{2}}\right\}^{-1}\right.
\end{aligned}
$$

Approximations, Eqs. (12) and (13), are very simple in form. Thus, they are proper for signal transformation and make it possible to realize the flow measurement in real time with the aid of analog electronic circuits. The term numbers of the firstorder lag elements and the second-order lag elements in the approximations depend respectively on the required low-frequency and high-frequency accuracy.

Figure 3 and Fig. 4 show the frequency characteristics of $M_{1}$, and $M_{2}$. It is clear that the exact models and their approximations get closer and closer in magnitude and phase with the increase of the term number of the second-order lag elements. Both of the approximate models $M_{1}$ and $M_{2}(m=4, n=10)$ are highly precise over a wide frequency range ${ }^{(7)}$.

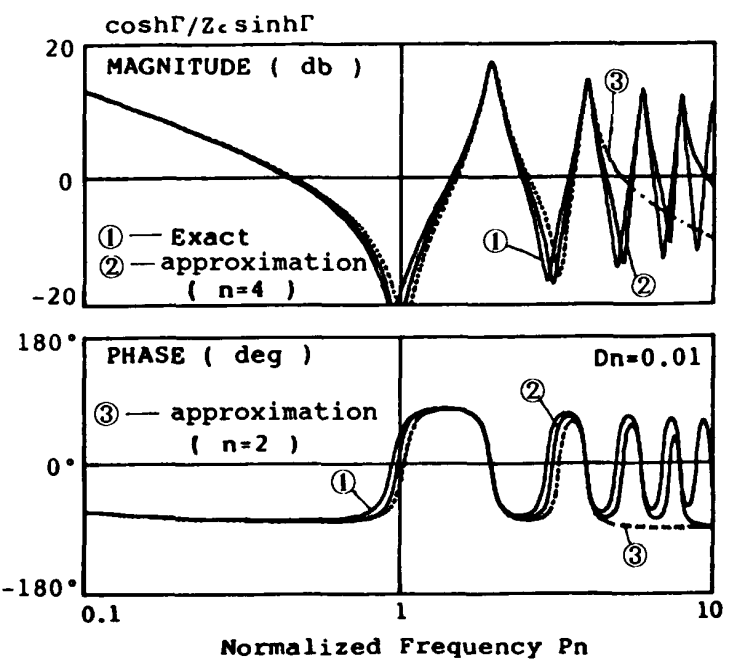

Fig. 3 Frequency characteristics of $M_{1}$

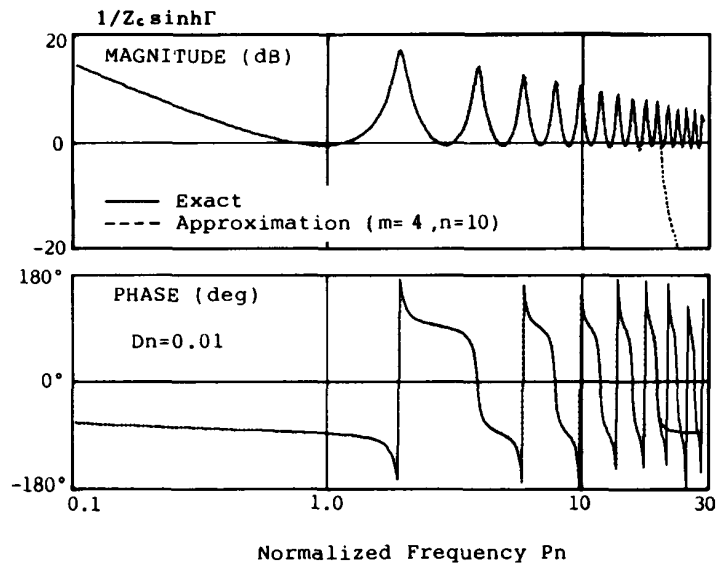

Fig. 4 Frequency characteristics of $M_{2}$

\section{Digital Transformation}

By means of the transfer relation in Fig.2, unsteady flow rates $Q_{1}$ and $Q_{2}$ can be calculated from the pressure signals $P_{1}$ and $P_{2}$. The signal transformation methods are as follows.

(1) convolution integral calculus.

(2) FFT method.

(3) characteristics method.

(4) recursive calculus.

(5) digital filter.

Each of these methods has its own advantages. For example, the FFT method is very effective for the measurement of pulsating flow rate with constant frequency components ${ }^{(6)}$. In addition, recursive calculus based on the approximate model, which is simple, reliable and applicable to the measurement of complicated flow, even nonperiodic random flow rate, is recommended in order to promote the efficiency of the signal transformation, and it provides a rational algorithm for the design of operation circuits to carry out the signal transformation in real time ${ }^{(8),(9)}$.

\section{Self-Check Function of Flow Measuring System}

As is well known, how to examine the exactness of measured results of unsteady flow rate is a difficult problem remaining to be solved because there still exists no calibration setup for the measurement in present techniques. On the other hand, the effects on the measurement, caused by environmental change, are difficult to avoid. Hence, monitoring should be performed in full time to obtain accurate flow rate results. To use LDA is one promising way to achieve the above goal. However, with the numerous troublesome problems to be solved in the method, development of a simple examining method for flow measurement becomes very important. For these reasons, a simple and reliable self-check method for flow measurement is put forward in this paper.

The principle of the self-check for flow measurement is shown in Fig. 5. Considering the cross sections 1 and 2 as the measuring sections, pressure $P_{1}$ and $P_{2}$ are primary signals and $P_{m}$ of the cross section in the middle of the two measuring sections is taken as the comparison signal (examining signal). The middle section, as shown in Fig. 5, divides the test pipe into two parts, where $L_{1}=L_{2}=L / 2$. Hence, the dimensionless dissipation number: $D_{n 1}=D_{n 2}=D_{n 3} / 2$. Combining and arranging the transfer matrix equations for the pipes $L_{1}$, and $L_{2}$ gives the following two equations:

$$
\begin{aligned}
& \hat{P}_{m}\left(s^{*}\right)=\frac{1}{\cosh (\Gamma / 2)} \frac{\hat{P}_{1}+\hat{P}_{2}}{2} \\
& \hat{P}_{m}\left(s^{*}\right)=\frac{Z_{c}}{\sinh (\Gamma / 2)} \frac{\hat{Q}_{1}-\hat{Q}_{2}}{2}
\end{aligned}
$$


The accurate approximations of $1 / \cosh \Gamma$ and $z_{c} / \sinh$ $\Gamma$ can be obtained by means of a previous technique $^{(10)}$.

$$
\begin{aligned}
& \frac{1}{\cosh \Gamma} \approx \sum_{i=1}^{n} \frac{a_{3 i} s^{*}+b_{3 i}}{s^{* 2}+2 \zeta_{i} \omega_{n i} s^{*}+\omega_{n i}^{2}} \\
& \frac{Z_{c}^{\prime}}{\sinh \Gamma} \approx \frac{1}{D_{n} s^{*}}+\sum_{i=1}^{n} \frac{a_{l i} s^{*}+b_{l i}}{s^{* 2}+2 \zeta_{i} \omega_{n i} s^{*}+\omega_{n i}^{2}}
\end{aligned}
$$

In a praotical flow measuring system, the pressure signals $P_{1}, P_{2}$ and $P_{m}$ can be simultaneously measured by pressure transducers. Then the measured signal $P_{m}$ is compared with $P_{m}$ (output) calculated from the primary signals $P_{1}$ and $P_{2}$ to judge whether or not the flow rates $Q_{1}$, and $Q_{2}$ calculated from $P_{1}$ and $P_{2}$ are accurate. Such an examining method, which confirms whether or not the transfer relations of the fluid variables in actual a pipe flow belong in the axial symmetry laminar flow, is called a "self-check function". In other words, the flow rates $Q_{1}$ and $Q_{2}$ calculated from $P_{1}$ and $P_{2}$ may be trusted only when the actual signal $P_{m}$ and calculated $P_{m}$ (output) agree precisely with each other because it suggests that the transfer characteristics of the fluid variables in actual flow may be expressed as the transfer matrix (Eq. ( 5 )).

\section{Measuring System}

In the above sections, a measurement of unsteady flow rate and its self-check principle have been introduced. According to the dual-pressure method, a measuring system is designed as shown schematically in Fig. 6. The test pipe is $11.7 \mathrm{~mm}$ in diameter and 6 . $4 \mathrm{~m}$ in length. The upstream end of the pipe is connected to a hydraulic power source. The downstream end is connected to a pulsating flow generator ${ }^{(11)}$, which is driven by a DC motor with an accurate speed regulator. The generator is composed of a fixed fluid damper $R_{1}$ and an adjustable orifice $R_{2}$ with an on-off rotary valve. The steady component and the amplitude of the pulsating flow are determined by $R_{1}$ and $R_{2}$, and the pulsating frequency is determined by the

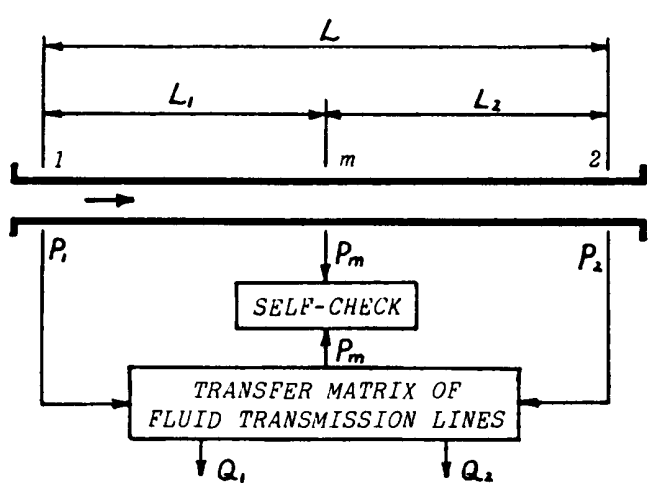

Fig. 5 Principle of self-check method for fluid measurement motor speed. The fluid in the pipe is hydraulic oil. The pressure signals at the cross sections 1,2 and $\mathrm{m}$ are measured by three pressure transducers. The amplified signals are fed to a 12 -bit $A / D$ converter. Then they are sent to a micro-computer (PC9801) for signal analysis and saved on a hard disk. For comparison, the recursive method and the characteristics method are adopted in the digital transformation of the fluid signals.

\section{Experimental Results and Considerations}

In order to ensure the precision of the system, it is necessary for three amplified pressure signals $P_{1}, P_{2}$ and $P_{m}$, dependent on the pressure transducers and their amplifiers, to have the same gain characteristics. For this reason, it is confirmed first that the pressure drops $\left(P_{1}-P_{2}\right),\left(P_{1}-P_{m}\right)$ and $\left(P_{m}-P_{2}\right)$ along the pipe are proportional to the flow rate. Then the transfer relations of the pressure signals are examined over a wide frequency range. As stated above, by the use of the recursive calculus based on the transfer function (Eq. (25)), $P_{m}$ (output) at the middle of the pipe can be acquired from $P_{1}$ and $P_{2}$. Figure 7 shows an illustration of the measured results in the frequency $f=300$ $\mathrm{Hz}$. It is found that the calculated $P_{m}$ (output) agrees well with the actual $P_{m}$. It should be mentioned that measuring $P_{m}$ has no other special significance other than confirming that the actual pipe flow observes the fluid transmission line dynamics. Therefore, flow rates $Q_{1}$ and $Q_{2}$ calculated by the use of the same method should also be authentic.

When the pulsating flow generator is closed, the measured results of the fluid transients caused by a quick opening of the check valve at the upstream area are shown in Fig.8. As shown in the figure, not only is the agreement between the $P_{m}$ (output) calculated from $P_{1}, P_{2}$ and measured $P_{m}$ is excellent, but also it was confirmed, as expected, that the flow rate $Q_{1}$, passing the cross section 1 converged to zero with damped oscillation, and $Q_{2}$ of the cross section 2 near the closed generator is small enough to be regarded as negligible.

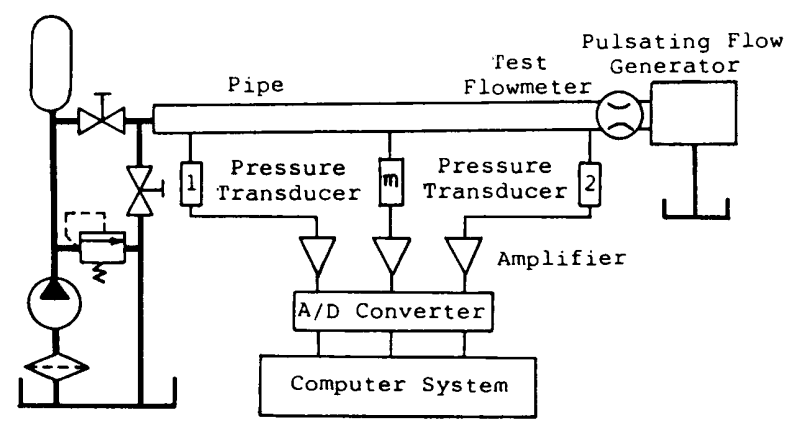

Fig. 6 Measuring system for unsteady flow rate 
One of the measured results of the pulsating flow rate is shown in Fig. 9. Figures 9 (f) and (e) show respectively the measured input signals $P_{1}$ and $P_{2}$, and Fig. 9 (d) demonstrates the comparison of the pressure signal $P_{m}$ calculated from $P_{1}$ and $P_{2}$ with the measured $P_{m}$. Figures 9 (a), (b) and (c) show,
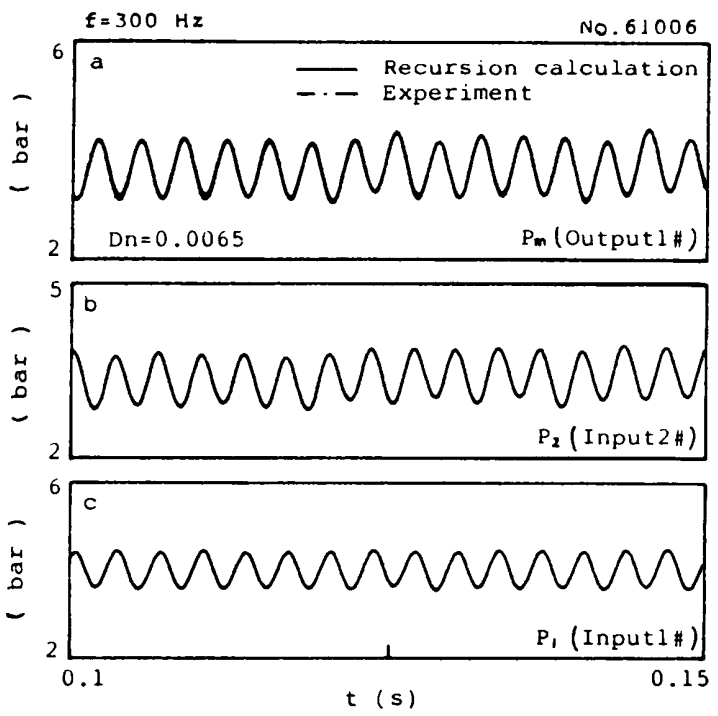

Fig. 7 Measured result of self-check signal
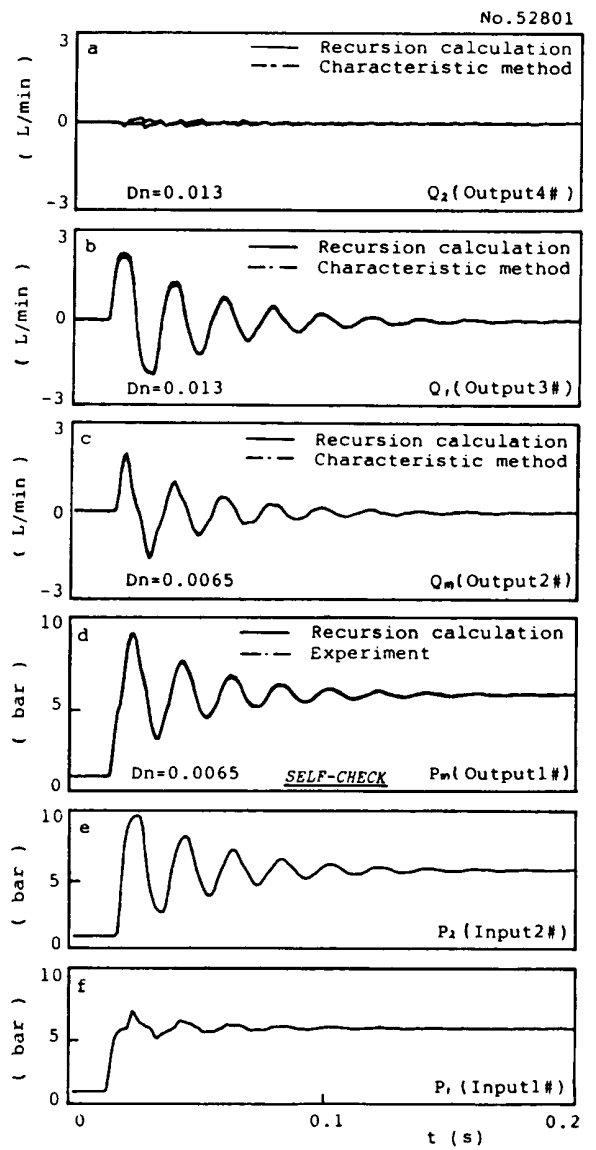

Fig. 8 Measured results of fluid signals (Step input) respectively, the flow rates $Q_{1}, Q_{2}$ and $Q_{m}$ obtained from $P_{1}$ and $P_{2}$ by the transfer relations. From the numerical calculated results by the characteristics method as well as the comparison of both signals $P_{m}$, it can be confirmed that the flow rates obtained by the measurement proposed in this paper are accurate and reliable. The frequency characteristics of the measured results are illustrated in Figs. 10 and 11, whose test frequency range is from $0 \mathrm{~Hz}$ to $300 \mathrm{~Hz}$. Amplitude and phase characteristics for the input and the output fluid signals are all calculated through the FFT program. In application, if pressure at an end of the pipe may be regarded as a constant, fluctuations of flow rate $Q_{1}$ and $Q_{2}$ can be obtained by another pressure signal only. The frequency characteristics of the measured results in the above case are shown in Fig. 12.

As shown in these figures, the experimental results agree well with the theoretical values in both amplitude and phase characteristics.

\section{Conclusions}

In this paper, a measuring system with a selfcheck function of high-frequency pulsating flow rate
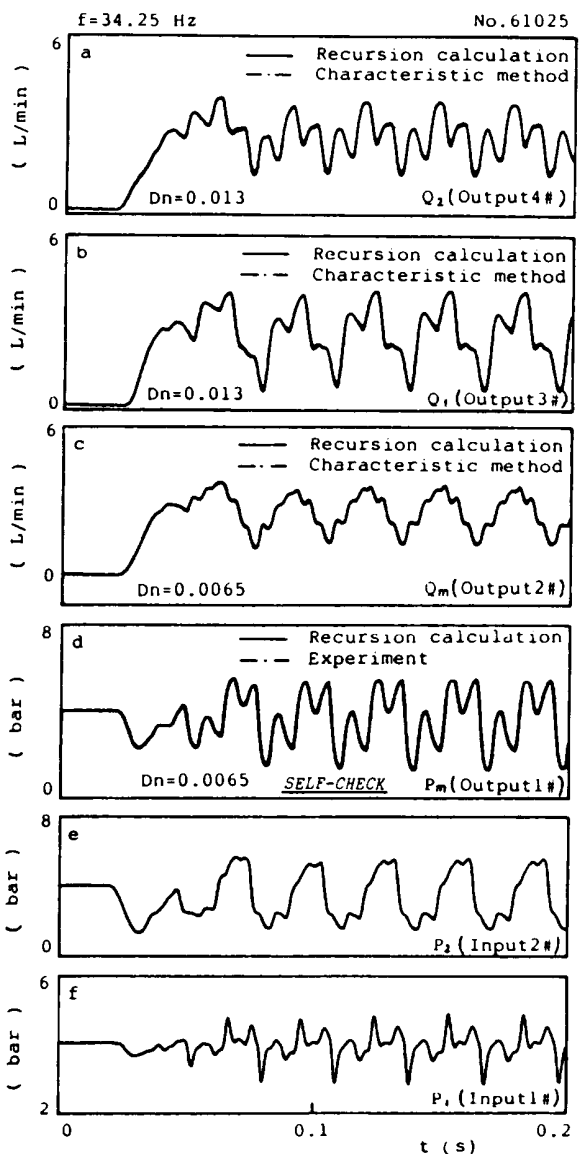

Fig. 9 Measured results of pulsating flow rate 

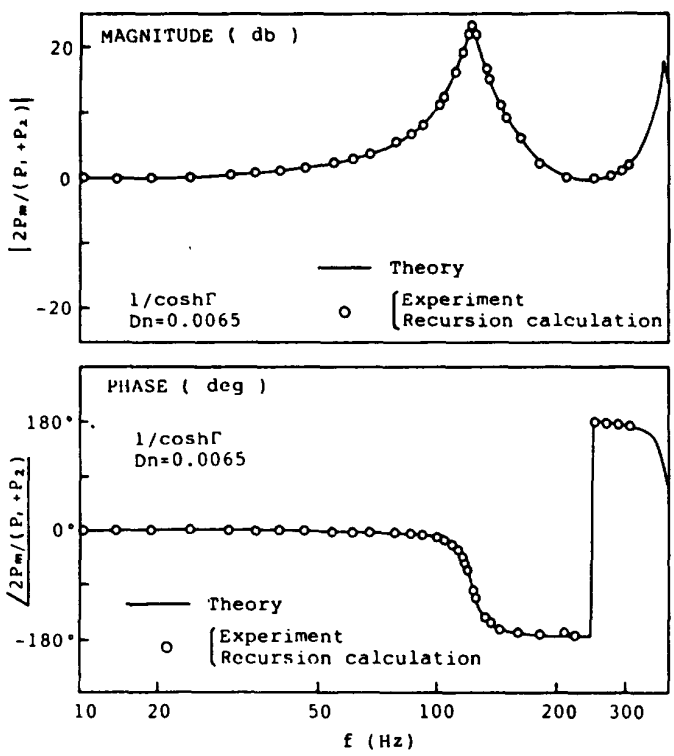

Fig. 10 Frequency characteristics of measured results

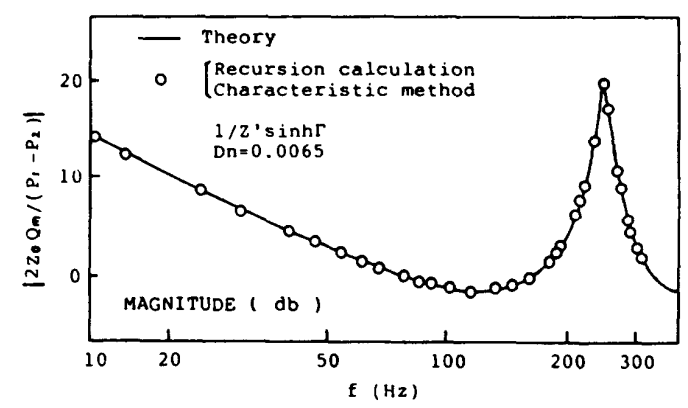

Fig. 11 Frequency characteristics of measured results

was set up by means of the dual-pressure method. The system was investigated and validated by analysis and experiments to confirm its conspicuous advantages as follows :

(1) Only a hydraulic pipe, without using any special resistances as choke or orifice, is applied to flow measurement in the method. Therefore, such measurement does not destroy the original flow state, and superfluous pressure loss can be decreased.

(2) This setup, not having any mechanical motion parts, is of good dynamic characteristics in the fluid measurement.

(3) The measuring system has made it convenient to measure various complex unsteady flow rates, such as nonperiodic flow or high-frequency pulsating flow.

(4) The mathematical models for the signal transformation are the linear combination of the simplex terms, so their transfer characteristics can be reproduced by easily assembled analog electronic circuits or digital filters. That is, the models make it possible to finish the signal transformation in real
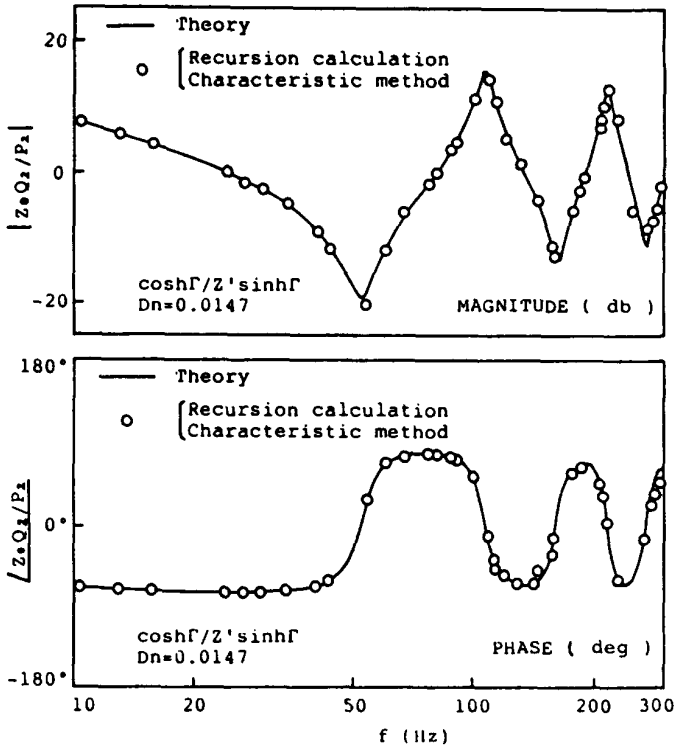

Fig. 12 Frequency characteristics of measured results time.

(5) The system can examine by its self-check function whether or not the measured results of flow rate are accurate and reliable, without using any other fluid measurement or flowmeters. Hence, it can also be used as a comparison standard for evaluating other flowmeters or measuring methods.

The measurement is not only simple but also effective and practical. It provides an important measuring means for studying the dynamic characteristics of hydraulic components or systems and for developing a new instantaneous flowmeter.

\section{Acknowledgments}

We express our gratitude to Professor TAKENAKA (Musashi Institute of Technology, JAPAN) and Professor KITAGAWA (Tokyo Institute of Technology, JAPAN) for the helpful advice given to this research. The research work was supported by the "FOK YING TUNG Education Foundation", the State Committee of Education, CHINA.

\section{References}

(1) Kitagawa, A. and Zhao T., Development of Measuring Technique of Unsteady Flow Rate (1st), Jpn. Instrumentation and Automation, (in Japanese), Vol. 15, No. 8 (1987), p. 192.

(2) Zhao, T., Kitagawa, A. and Takenaka, T., A Real Time Measuring Method of Unsteady Flow Rate Employing Centerline Velocity in a Pipe, JSME International Journal, Vol. 30, No. 263 (1987), p. 767. ( Trans. of JSME, Vol. 52, No. 482, B (1986), p. 3484.)

( 3 ) Zhao, T., Kitagawa, A., Kagawa, T. and Takenaka, T., A Real Time Measuring Method of Un- 
steady Flow Rate and Velocity Employing Differential Pressure in a Pipe, JSME International Journal, Vol. 30, No. 260 (1987), p. 263. (Trans. of JSME, Vol. 52, No. 480, B(1986), p. 2851.)

(4) Zhao, T., Sanada, K., Kitagawa, A. and Takenaka, T., Real Time Measurement of High Frequency Pulsating Flow Rate in a Pipe, Journal of Dynamic System, Measurement \& Control, Trans. of ASME, Vol. 112, No. 4 (Dec.1990), p. 762.

( 5 ) Zhao, T., Sanada, K., Kitagawa, A. and Takenaka,T., A Study on Measurement Accuracy of a Method of Measuring Unsteady Flow Rate Employing a Differential Pressure in a Pipe, Trans. of JSME, (in Japanese), Vol. 54, No. 503, B(1988), p. 1649.

(6) Zhao, T., Peng, G. Z. and Xu, Y. M., A Study on Calibration Method of Dynamic Flow Rate, Proceedings of the 2nd International Conferences on Fluid Power Transmission and Control, Hangzhou, CHINA, (Mar.1989), p. 741.
( 7 ) Zhao, T., Peng, G.Z. and Xu, Y. M., Practical and Accurate Approximate Method for Distributed Parameter Pipe Model, Hydraulics \& Pneumatics, Trans. of CHPS, (in Chinese), Vol. 53, (1990), p. 9.

(8) Zhao, T., Transformation Calculus of the Variables in an Unsteady Pipe Flow, Trans. of JSME, (in Japanese), Vol. 51, No. 472, B(1985), p. 4139.

(9) Luo, S. W., Zhao, T. and Lu, W. H., Recursive Calculus for Simulation of Pipe-volume System, Machine Tool \& Hydraulics, (in Chinese), Vol. 105, CSME (1990), p. 31.

(10) Zhao, T., Peng, G. Z. and Xu, Y. M., Approximate Models of Pipe Dynamics Considering Unsteady Viscosity Loss, Jpn. Science of Machine, (in Japanese), Vol. 42, No. 11, (Nov. 1990), p. 1229.

(11) Peng, G. Z., Zhao, T. and Xu, Y. M., A Calibration Setup for Instantaneous Flowmeters, Proceedings of the International Conference on Fluid Dynamic Measurement and its Applications, Beijing, China, (Oct. 1989), p. 412. 